\title{
Wavelet-based Methods for Clutter Removal from Radar Wind Profiler Data
}

\author{
Lutz A. Justen $^{a}$, Gerd Teschke ${ }^{a}$ and Volker Lehmann ${ }^{b}$ \\ ${ }^{a}$ Zentrum für Technomathematik, University of Bremen, \\ Bibliothekstr. 1, 28359 Bremen, Germany \\ ${ }^{b}$ Deutscher Wetterdienst, Am Observatorium 12, 15848 Tauche OT Lindenberg, Germany
}

\begin{abstract}
The common way to process radar wind profiler (RWP) data by moments estimation of the Fourier power spectrum fails in presence of transient intermittent clutter contributions. Wavelets are especially suitable for detecting and removing transient components because of their high localization in time and frequency domain. We give an overview on the wavelet filtering of contaminated discrete RWP signals and introduce a new technique involving the wavelet packet decomposition and a splitting in progressive and regressive signal components. This technique has been successfully tested on severely real-data sets where classical wavelet routines fail.
\end{abstract}

Keywords: Wavelets, wavelet packets, intermittent clutter filtering, radar wind profiler, signal processing, time-frequency decomposition

\section{INTRODUCTION}

In recent years, wavelet analysis has become a very powerful tool in applied mathematics. The first applications of wavelets were concerned with problems in image/signal analysis/compression. Furthermore, quite recently, wavelet algorithms have also been applied very successfully in numerical analysis, geophysics, meteorology, astrophysics and in many other fields. Especially, it has turned out that the specific features of wavelets can also be efficiently used for certain problems in the context of radar signal analysis. The aim of this paper is to give an overview on the analysis of discrete RWP data and to explain how wavelets can be utilized for clutter removal.

The goal of RWP systems is to gather information concerning the three dimensional atmospheric wind vector. Radio frequency pulses are emitted and backscattered from small inhomogeneities in the atmosphere. The reflected signal is sampled at certain rates corresponding to different heights. The Doppler shift of the atmospheric (clear-air) signal, which can assumed to be constant over the small measurement period (quasistationarity), generates a peak in the Fourier power spectrum. The classical analysis consists of the following steps: coherent integration, Fourier analysis, spectral moment estimation, and, finally, the wind estimation.

However, intermittent clutter contributions such as erroneous bird/airplane reflections are well known to be non-stationary (transient), even over short time scales. ${ }^{1-3}$ As a matter of fact, Fourier transformation cannot resolve transient frequencies. Moment estimation will necessarily fail in these cases. This is exactly the topic where wavelet analysis suggests itself since wavelets are by construction very localized functions being able to resolve frequency transitions during short time periods. Indeed, several wavelet de-noising algorithms have already been successfully applied. ${ }^{4,5}$ It is especially noteworthy that these wavelet algorithms are numerically very efficient can be executed on-line on modern PCs.

The clutter removal process consists of three steps.

- First of all, the signal is decomposed into a wavelet series by means of a fast wavelet transform. Since the wavelet coefficients contain time as well as frequency information, one speaks of a time-frequency (TF) decomposition.

Further author information:

L.A.J.: E-mail: justen@math.uni-bremen.de, Telephone: +494212184458 
- In a second step, a thresholding procedure is applied to identify and remove intermittent clutter from the TF decomposition.

- Finally, the cleared signal is reconstructed from the filtered wavelet coefficients. The Doppler shift can now be estimated directly from the cleared Fourier powerspectrum.

The crucial point of the wavelet decomposition is the separation of clutter and clear-air signal. That is, there must be a certain set of wavelet coefficients containing only clutter information and another set containing only clear-air information. If these sets can be identified and do not intersect, removal of the clutter set will lead to a well filtered reconstructed signal which admits a wind estimation by its Fourier spectrum. Hence, the wavelet decomposition should keep each set as small as possible to reduce the risk of intersection.

In Section 2, we will briefly describe how the classical fast dyadic wavelet transform works. Special emphasis is set on TF localization properties and the separability of clear-air signal and clutter. We will see in Sections 3 and 4 how the separability can be dramatically improved by splitting the signal into progressive and regressive parts and afterwards decomposing each part by means of the wavelet packet decomposition. For proofs and more background information on wavelets in general and wavelet packets we refer to the book of Mallat. ${ }^{6}$ Finally, we choose an adaptive threshold for clutter removal (Section 5) and give some real-data examples (Section 6).

\section{DISCRETE WAVELET TRANSFORM}

Assume we have a sequence of discrete values $a^{0}=\left(a_{n}^{0}\right)$ sampled from a continuous function $f \in \mathbb{L}^{2}(\mathbb{R})$ at intervals of unity length, i.e. $a_{n}^{0}=f(n), n \in \mathbb{Z}$. A discrete wavelet transform step decomposes the sequence $a^{0}$ into two sequences $a^{1}$ and $d^{1}$ by means of a low-pass filter $h=\left(h_{n}\right)$ and a high-pass filter $g=\left(g_{n}\right)$ followed by a downsampling of order 2 . The sequence $a^{1}$ is called approximation and contains low-frequency information of $a^{0}$. The sequence $d^{1}$ is said to be the details and contains high-frequency information. $h$ and $g$ are called decomposition filters. They are usually real and can be obtained from many textbooks about wavelet analysis, see e.g. Mallat. ${ }^{6}$ The filtering process can be written as

$$
\begin{aligned}
& a^{1}=\left(a_{n}^{1}\right)=\left(a^{0} * h\right) \downarrow 2 \\
& d^{1}=\left(d_{n}^{1}\right)=\left(a^{0} * g\right) \downarrow 2,
\end{aligned}
$$

where the convolution $x * y$ of two sequences $x$ and $y$ is given by $(x * y)_{n}=\sum_{k \in \mathbb{Z}} x_{k} y_{n-k}$. The downsampling operator $\downarrow p, p \in \mathbb{N}$, maps a sequence $\left(x_{n}\right)$ to the sequence $\left(y_{n}\right)=\left(x_{p n}\right)$, i.e. only every p-th sample is kept. $a^{0}$ can be reconstructed from $a^{1}$ and $d^{1}$ by means of the reconstruction filters $\tilde{h}$ and $\tilde{g}$ and upsampling:

$$
f=\left(a^{1} \uparrow 2\right) * \tilde{h}+\left(d^{1} \uparrow 2\right) * \tilde{g} .
$$

The upsampling operator $\uparrow p$ inserts zeros into a sequence $x$, i.e.

$$
(x \uparrow p)_{n}= \begin{cases}x_{q} & \text { if } n / p=q \in \mathbb{Z} \\ 0 & \text { if } n / p \notin \mathbb{Z} .\end{cases}
$$

A discrete wavelet transform (DWT) of level $J$ decomposes the sequence $a^{0}$ into $J$ details $d^{1}, \ldots, d^{J}$ and one approximation $a^{J}$ by successively applying the given filter bank algorithm to the approximations. See Figures 1 and 2 for a complete description of level 3 decomposition and reconstruction trees.

In a numerical implementation, a sequence of finite length is convoluted with filters $h$ and $g$, which are also of finite length*. For the convolution to be well-defined, the signal has to be extended (padded) at both ends e.g. by zeros or periodically. The downsampling then (approximately) halves the number of coefficients in each sequence, such that the total number of coefficients is kept. Hence, one speaks of a non-redundant transformation. Since only $O(N)$ operations are needed for decomposition and reconstruction, where $N$ is the number of samples in $a^{0}$, the algorithm is called fast wavelet transform.

\footnotetext{
${ }^{*}$ One talks of finite impulse response (FIR) filters
} 


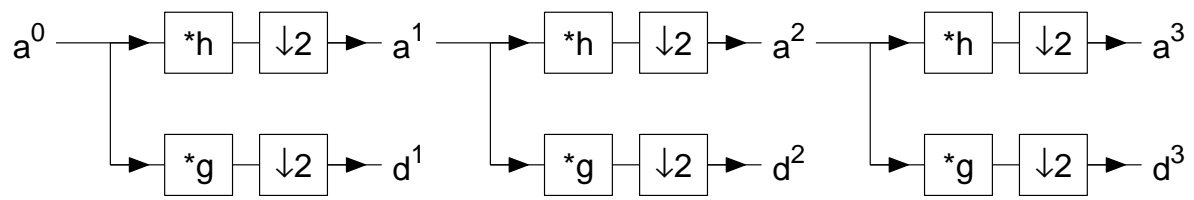

Figure 1. 3 levels of a DWT decomposition filter bank algorithm. The sequence $a^{0}$ is split into details $d^{1}, d^{2}$ and $d^{3}$ and an approximation $a^{3}$

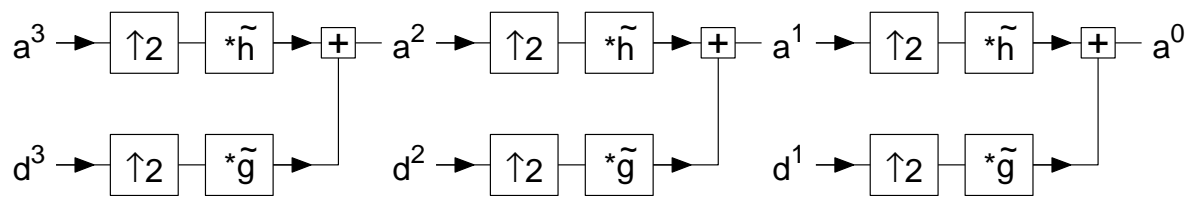

Figure 2. 3 levels of a DWT reconstruction filter bank algorithm. The sequence $a^{0}$ is reconstructed from details $d^{1}, d^{2}$ and $d^{3}$ and an approximation $a^{3}$.

\subsection{Time-Frequency Localization Properties}

For the wavelet analysis of RWP signals it is of prime importance to know how well certain features can be resolved in time as well as in frequency. In order to get more insight into these time-frequency localization properties, it is instructive to look at the sequences of coefficients in Fourier domain. The Fourier transform $\hat{x}=\mathcal{F}(x)$ of a sequence $x$ is defined by

$$
\hat{x}(\omega)=\mathcal{F}(x)(\omega)=\sum_{n \in \mathbb{Z}} x_{n} \exp (-i \omega n) .
$$

For signals sampled at a time spacing of $1, \omega=\pi$ is the Nyquist frequency, i.e. the largest frequency which can be represented without aliasing effects. From the filter bank algorithm described in Figure 1 we know that the sequences of coefficients can be written as

$$
\begin{aligned}
& \left.\left.\left.\left.\left.\left.a^{j}=\left(a^{j-1} * h\right) \downarrow 2=\left(\cdots\left(a^{0} * h\right) \downarrow 2\right) * h\right) \downarrow 2\right) \cdots\right) * h\right) \downarrow 2\right) * h\right) \downarrow 2 \\
& \left.d^{j}=\left(a^{j-1} * g\right) \downarrow 2=(\cdots(a^{0} \underbrace{* h) \downarrow 2}) \underbrace{* h) \downarrow 2}_{j \text { times }}) \cdots\right) \underbrace{* h) \downarrow 2}) \underbrace{* g) \downarrow 2} .
\end{aligned}
$$

The Fourier transforms of a downsampled sequence $x \downarrow 2$ and an upsampled sequence $x \uparrow 2$ are given by

$$
\begin{aligned}
& \widehat{x \downarrow 2}(\omega)=\frac{1}{2}(\hat{x}(\omega / 2)+\hat{x}(\omega / 2+\pi)) \\
& \widehat{x \uparrow 2}(\omega)=\hat{x}(2 \omega) .
\end{aligned}
$$

By taking the Fourier transform on both sides of Equations (3) and (4) and using the convolution theorem $\widehat{x * y}=\hat{x} \cdot \hat{y}$ we thus obtain by induction

$$
\begin{aligned}
& a^{j}=\left(a^{0} * h *(h \uparrow 2) * \cdots *\left(h \uparrow 2^{j-2}\right) *\left(h \uparrow 2^{j-1}\right)\right) \downarrow 2^{j} \\
& d^{j}=\left(a^{0} * h *(h \uparrow 2) * \cdots *\left(h \uparrow 2^{j-2}\right) *\left(g \uparrow 2^{j-1}\right)\right) \downarrow 2^{j} .
\end{aligned}
$$

If we denote the inverse Fourier transform by

$$
x(t)=\mathcal{F}^{-1}(\hat{x})=\frac{1}{2 \pi} \int_{-\pi}^{\pi} \hat{x}(\omega) \exp (i \omega t) \mathrm{d} \omega,
$$


we may express Equations (7) and (8) also as

$$
\begin{aligned}
a^{j} & =\left(\mathcal{F}^{-1}\left(\hat{a}^{0} \cdot \prod_{p=0}^{j-1} \hat{h}\left(2^{p} \cdot\right)\right)\right) \downarrow 2^{j} \\
d^{j} & =\left(\mathcal{F}^{-1}\left(\hat{a}^{0} \cdot \prod_{p=0}^{j-2} \hat{h}\left(2^{p} \cdot\right) \hat{g}\left(2^{j-1} \cdot\right)\right)\right) \downarrow 2^{j} .
\end{aligned}
$$

We posed the question which information of $a^{0}$ is contained in the wavelet coefficients $a^{j}$. Equation (9) answers this question. In Fourier domain, $\hat{a}^{0}$ is multiplied by a product of Fourier transformed and scaled low-pass filters of the form $\hat{h}\left(2^{p}\right.$.). Figure 3 (left) shows plots of $|\hat{h}|^{2}$ for several wavelet types. Wavelets are always constructed such that the energy of $\hat{h}$ is concentrated in the interval $[-\pi / 2, \pi / 2]$, which justifies the name low-pass filter. Hence, $\hat{h}(\omega) \cdot \hat{h}(2 \omega)$ is concentrated in $[-\pi / 4, \pi / 4]$ and so on. Multiplying $\hat{a}^{0}$ with the product filter, only energy at frequencies $\omega \in\left[-\pi / 2^{j}, \pi / 2^{j}\right]$ is kept. Any energy of $a^{0}$ at different frequencies is cut off. The final downsampling does not change this fact.

The high-pass filter $g$ is complementary to $h$. Its energy is concentrated in $[-\pi,-\pi / 2] \cup[\pi / 2, \pi]$. Repeating the above analysis for $d^{j}$ shows that $d^{j}$ contains information of $a^{0}$ at frequencies concentrated in $\left[-\pi / 2^{j-1},-\pi / 2^{j}\right] \cup$ $\left[\pi / 2^{j}, \pi / 2^{j-1}\right]$.

Figure 3 (right) illustrates these results in TF domain. The detail coefficients $d^{1}=\left(a^{0} * g\right) \downarrow 2$ are well localized in time since the high-pass filter $g$ consists of only a few non-zero coefficients for most commonly used wavelets. But frequency localization is rather bad since $d^{1}$ covers a large amount of the frequency axis. The opposite is true for $a^{3}$, where time localization is poor due to the large width of $h *(h \uparrow 2) *(h \uparrow 4)$, but frequency localization is better. Also note that all coefficients contain symmetrically negative as well as positive frequencies. Hence, we cannot distinguish between positive and negative frequencies. In Section 4 we will show how to overcome this problem.
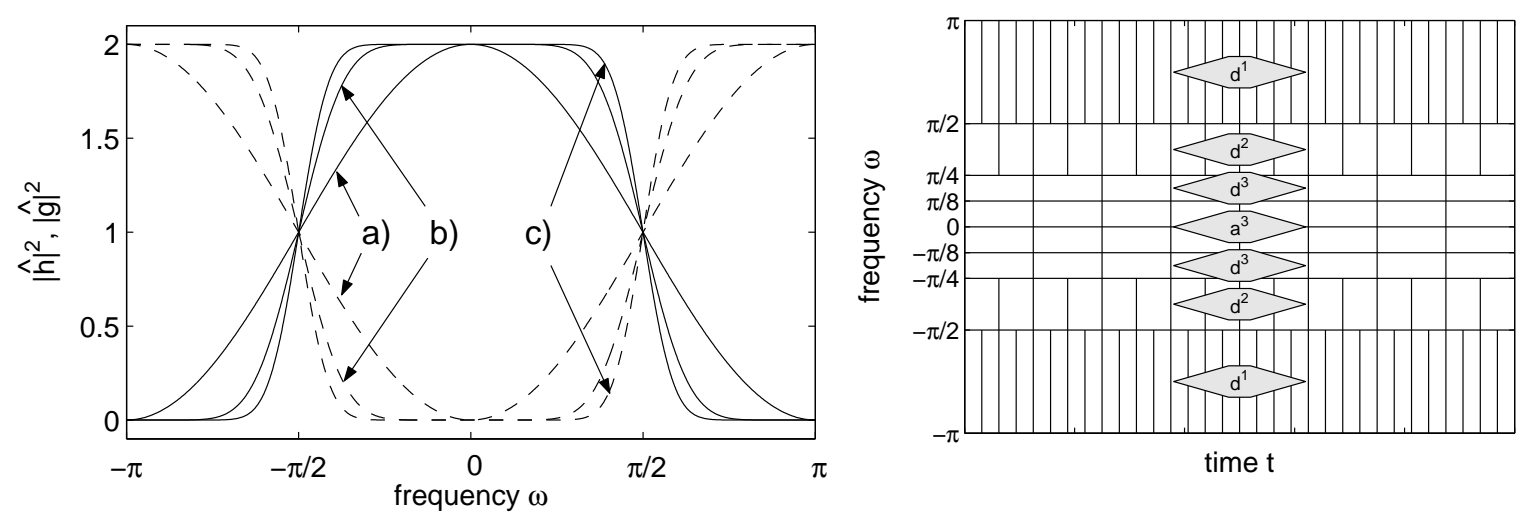

Figure 3. Left: 3 decomposition filter pairs $|\hat{h}|^{2}$ (solid) and $|\hat{g}|^{2}$ (dashed). a) Haar (length 2), b) Daubechies db5 (length 10), c) Coifman coif5 (length 30). Right: Schematic overview of the TF coverage of the wavelet coefficients of a level 3 DWT.

\subsection{Artificial RWP Signal}

Let us present an example where we compute the DWT of a pretty much simplified artificial RWP-like signal. The signal shall contain a stationary clear-air signal and a transient airplane echo. The clear-air signal is modeled by a plane wave $f_{\text {air }}=\exp \left(i \omega_{\text {air }} t\right)$, so no Gaussian random distribution is added to its frequency behavior for simplicity. In order to model the airplane echo, we undertake the following considerations:

- The airplane is more or less a point object, so there should only be one certain Doppler shift at each time, not a distribution of shifts. 
- When the airplane approaches the radar site, the Doppler shift is positive.

- When the airplane is right above the site or when its radial velocity component is zero, also the Doppler shift is zero.

- When the airplane leaves the site, the Doppler shift is negative.

A simple model for the behavior of the complex RWP signal is thus a function which linearly decreases its frequency. Such a function is called a linear chirp and is given by $f_{\text {clutter }}=\exp \left(-i \omega_{\text {clutter }} t^{2}\right)$. This model matches our observations surprisingly well, as we will see in Section 6. The total RWP signal is the sum of the two components, i.e.

$$
f(t)=f_{\text {air }}(t)+f_{\text {clutter }}(t)=\exp \left(i \omega_{\text {air }} t\right)+\exp \left(-i \omega_{\text {clutter }} t^{2}\right)
$$

We choose $\omega_{\text {air }}=\frac{3 \pi}{4}$ and $\omega_{\text {clutter }}=\frac{\pi}{2 \cdot 1024}$. By this choice, only $d^{1}$ is influenced by the clear-air signal and the airplane echo frequency exactly matches the Nyquist frequency at the end of the time interval. See Figure 4 for a plot of this signal and a schematic sketch of the TF behavior.
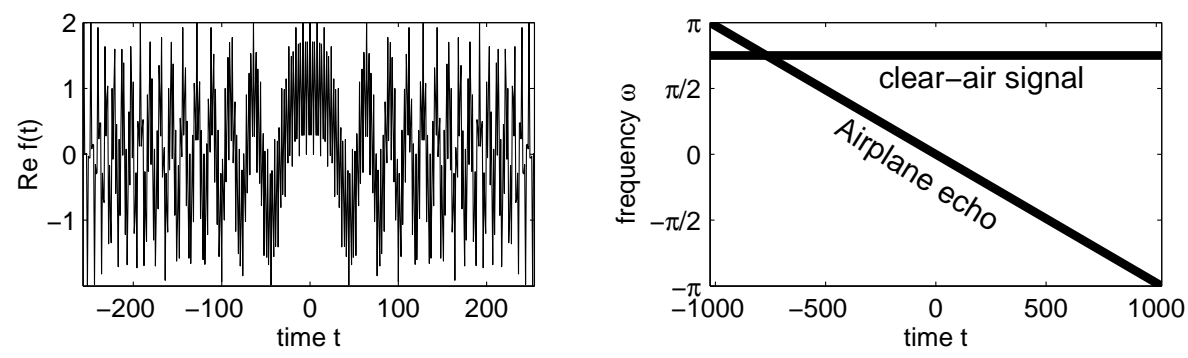

Figure 4. Left: Real part of the artificial RWP signal. Right: Schematic overview of the TF behavior.

After sampling $f$ at $t=-1024,-1023, \ldots, 1022,1023$ to obtain a complex signal vector $\left(f_{n}\right)$ of length 2048 , we compute a level 5 DWT using Daubechies db5 wavelet filters containing 10 non-zero coefficients for both $h$ and $g$. Figure 5 shows a plot of the squared absolute values of the wavelet coefficients.

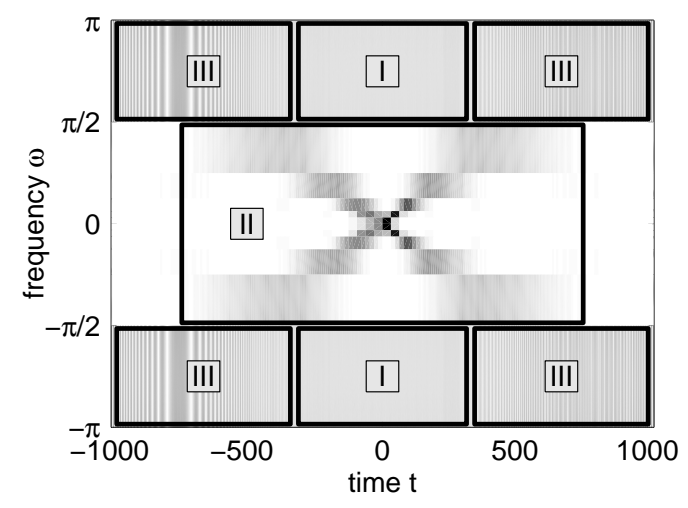

Figure 5. Level 5 DWT of $f$. Box I: clear-air information. Box 2: airplane echo. Box 3: Mixed clear-air signal and airplane echo. Compare also Figure 3.

Coefficients in boxes I contain clear-air information only and must not be removed in a thresholding process. Coefficients in box II contain airplane echo information only and can be filtered without influencing the clearair signal. Coefficients in boxes III contain mixed information of clutter and signal. A filtering routine would influence and possibly remove clutter as well as signal information. Note that boxes III cover a large part of the TF plane. 
As we said in the introduction, a TF decomposition should keep the set of coefficients containing both clutter and clear-air information (i.e. boxes III) as small as possible. Consequently, we need to look for more suitable methods to decompose the TF plane.

\section{WAVELET PACKETS}

We have seen in Section 2 that the DWT may not be suitable for the separation of intermittent clutter and clear-air signal. In cases of high wind speed and slowly-varying airplane echoes there might be many wavelet coefficients containing both clutter and signal information because of the poor frequency localization of the high-frequency detail coefficients.

A way to circumvent this problem is a decomposition of the detail coefficients. Up to now, only approximations have been split into higher level approximations and details (see Figure 1). A wavelet packet transform (WPT) of level $J$ decomposes in each of $J$ steps both approximation and detail coefficients creating a binary decomposition tree. In order to not mess up with the names, we rename the wavelet coefficients: We set $c_{0}^{0}=a^{0}$ and define

$$
\begin{aligned}
c_{2 k}^{j+1} & =\left(c_{k}^{j} * h\right) \downarrow 2 \\
c_{2 k+1}^{j+1} & =\left(c_{k}^{j} * g\right) \downarrow 2
\end{aligned}
$$

for $j=0, \ldots, J-1$ and $k=0, \ldots, 2^{j}-1$. Hence, at level $j$ there are $2^{j}$ coefficient vectors. See Figure 6 for the WPT binary decomposition tree. $c_{0}^{0}$ can be reconstructed from $c_{k}^{J}, k=0, \ldots, 2^{J}-1$, by essentially the same

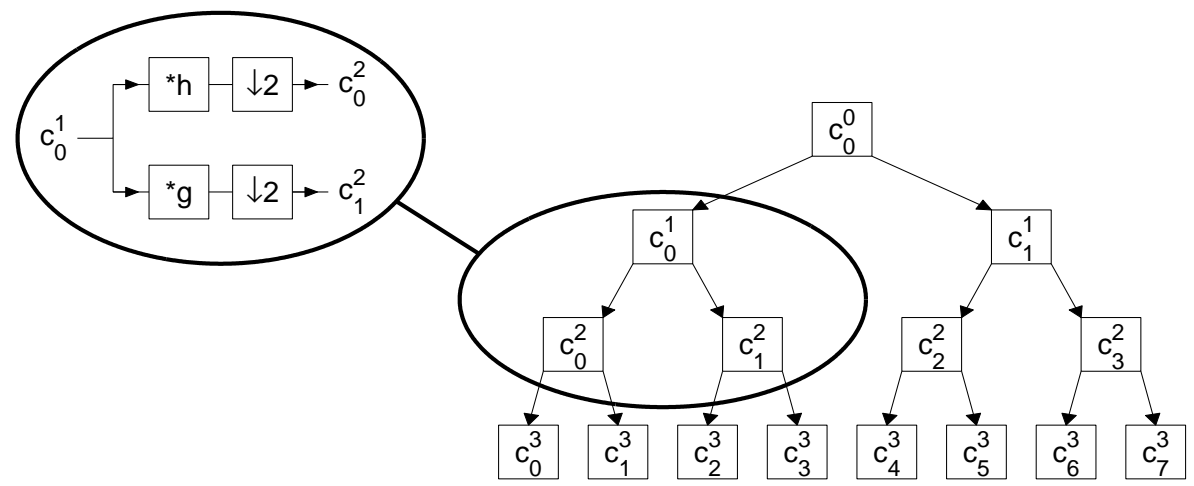

Figure 6. Wavelet packet binary tree. In each step, approximations as well as details are decomposed by a filter bank algorithm. Left children correspond to approximations and right children correspond to details.

means as for the DWT. Each decomposition step is inverted with the help of reconstruction filters $\tilde{h}$ and $\tilde{g}$ until $c_{0}^{0}$ is recovered.

The TF localization properties of the WPT are analyzed similarly to the DWT. Each leaf $c_{k}^{J}$ of the binary tree can be expressed as a convolution with upsampled filters followed by a downsampling. More precisely,

$$
c_{k}^{J}=\left(a^{0} * F_{1} *\left(F_{2} \uparrow 2\right) * \cdots *\left(F_{J} \uparrow 2^{J-1}\right)\right) \downarrow 2^{J},
$$

where $F_{n}$ has to be replaced by $h$ if the $\mathrm{n}$-th binary digit in $k$ is a zero and by $g$ if it is a one. For example, the binary digit representation of 5 is 101 . Hence, the sequence of filters is $g-h-g$ and $c_{5}^{3}=\left(a^{0} * g *(h \uparrow 2) *(g \uparrow 4)\right) \downarrow 8$. Knowing that $\hat{h}$ is most concentrated in $[-\pi / 2, \pi / 2]$ and that $\hat{g}$ is most concentrated in $[-\pi,-\pi / 2] \cup[\pi / 2, \pi]$, one can show that all of the possible $2^{J}$ combinations of filters $h$ and $g$ divide the frequency axis into intervals of equal size. Therefore, each leaf $c_{k}^{J}, k=0, \ldots, 2^{J}-1$, contains information of the original sequence $c_{0}^{0}$ that is equally well localized in frequency. The higher $J$ is, the better is this localization. The frequency intervals are given by

$$
I_{p}^{J}=\left[-(p+1) \pi 2^{-J},-p \pi 2^{-J}\right] \cup\left[p \pi 2^{-J},(p+1) \pi 2^{-J}\right] \quad, p=0, \ldots, 2^{J}-1 .
$$


$c_{k}^{J}$ contains information of $c_{0}^{0}$ at frequencies in $I_{G[k]}^{J}$, where the permutation $G$ can be recursively computed from

$$
\begin{aligned}
G[2 k] & = \begin{cases}2 G[k] & \text { if } G[k] \text { is even } \\
2 G[k]+1 & \text { if } G[k] \text { is odd }\end{cases} \\
G[2 k+1] & =\left\{\begin{array}{ll}
2 G[k]+1 & \text { if } G[k] \text { is even } \\
2 G[k] & \text { if } G[k] \text { is odd }
\end{array} .\right.
\end{aligned}
$$

Hence, the re-ordered set of vectors $\left(c_{G[k]}^{J}\right)_{k=0}^{2^{j}-1}$ is frequency-ordered.

Figure 7 (left) illustrates the TF coverage of the leaf vectors. A level 5 WPT of the artificial RWP signal using Daubechies db5 wavelet filters is shown in Figure 7 (right). Note that, in spite of some artifacts introduced by the WPT, the airplane echo can be expressed by considerably less wavelet coefficients than in the DWT-case. Also boxes III, where clutter and clear-air signal mix, are much smaller.
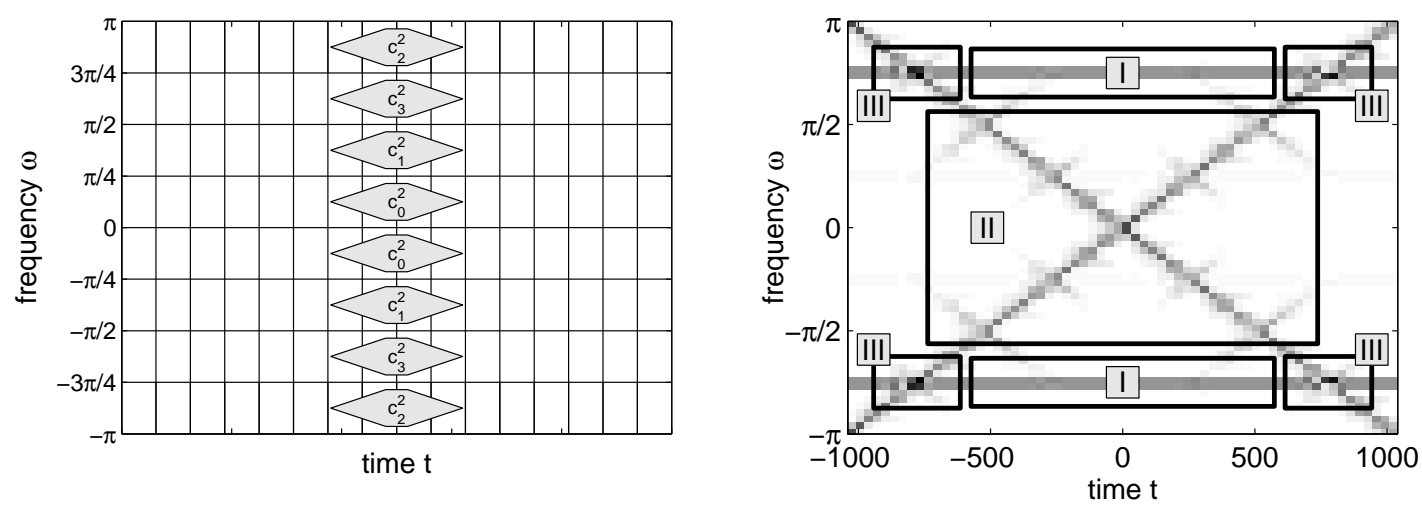

Figure 7. Left: Schematic overview of the TF coverage of the wavelet coefficients of a level 2 WPT. Note that the highest frequencies are covered by $c_{2}^{2}-$ not $c_{3}^{2}-$ due to the permutation $G[0,1,2,3]=(0,1,3,2)$. Right: Level 5 WPT of $f$. Box I: clear-air information. Box 2: airplane echo. Box 3: Mixed clear-air signal and airplane echo. The two signal components can be much better separated than for the DWT. Compare Figures 5 and 3.

But still, positive and negative frequencies cannot be distinguished. This is due to the fact that we have only considered real wavelet filters so far. For real wavelets, $|\hat{h}|$ and $|\hat{g}|$ are symmetric functions in $\omega$ covering parts of the positive frequency axis as well as the negative one. Next section, we shall show how to overcome this problem.

\section{POSITIVE AND NEGATIVE FREQUENCIES}

Consider the complex filters $h_{n}^{+}=i^{n} h_{n}$ and $h^{-}=(-i)^{n} h_{n} \cdot{ }^{7} \quad$ Since $\hat{h}$ is concentrated in $[-\pi / 2, \pi / 2]$, their Fourier transforms $\hat{h}^{+}$and $\hat{h}^{-}$are concentrated in $[0, \pi]$ and $[-\pi, 0]$, resp. We wish to make use of this property.

Before the WPT, we filter $c_{0}^{0}$ with $h^{+}$and $h^{-}$:

$$
{ }^{+} c_{0}^{0}:=c_{0}^{0} * h^{+} \quad \text { and } \quad-c_{0}^{0}:=c_{0}^{0} * h^{-} .
$$

${ }^{+} c_{0}^{0}$ contains information of $c_{0}^{0}$ at positive frequencies (progressive part) and ${ }^{-} c_{0}^{0}$ contains information of $c_{0}^{0}$ at negative frequencies (regressive part). ${ }^{+} c_{0}^{0}$ and ${ }^{-} c_{0}^{0}$ are then transformed by the WPT separately to obtain wavelet coefficients ${ }^{+} c_{k}^{J}$ and ${ }^{-} c_{k}^{J}$. The TF domain coverage of these coefficients is similar to last Section, but this time positive and negative frequencies can be separated, see Figure 8 (left).

Figure 8 (right) shows a plot of this frequency separated wavelet packet transform (FSWPT) of the artificial RWP signal. Airplane echo and clear-air signal overlap only on a very small number of coefficients and can be well separated. 

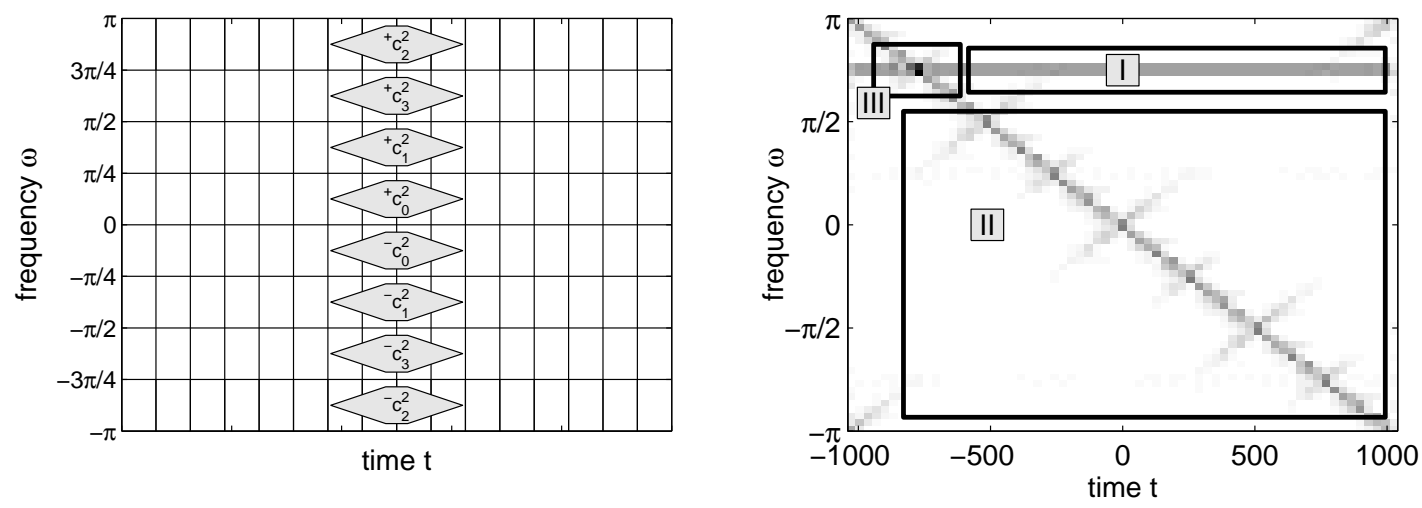

Figure 8. Left: Schematic overview of the TF coverage of the wavelet coefficients of a level 2 FSWPT. Right: Level 5 FSWPT of $f$. Box I: clear-air information. Box 2: airplane echo. Box 3: Mixed clear-air signal and airplane echo. The clear-air signal and airplane echo overlap is minimal. Compare also Figures 5 and 7.

Since the sequences ${ }^{+} c_{0}^{0}$ and ${ }^{-} c_{0}^{0}$ are not downsampled after the convolution with $h^{+}$and $h^{-}$, they contain twice as many samples as the original sequence $c_{0}^{0}$. Hence, the FSWPT is a redundant transformation with a 2:1 redundancy.

How can $c_{0}^{0}$ be reconstructed from ${ }^{+} c_{0}^{0}$ and ${ }^{-} c_{0}^{0}$ ? To answer this question, we make use of a basic property of the decomposition filter $h$ and the reconstruction filter $\tilde{h}$. They are related by

$$
\hat{h}(\omega) \hat{\tilde{h}}(\omega)+\hat{h}(\omega+\pi) \hat{\tilde{h}}(\omega+\pi)=2
$$

By defining $\tilde{h}_{n}^{+}:=i^{n} \tilde{h}_{n}$ and $\tilde{h}_{n}^{-}:=(-i)^{n} \tilde{h}_{n}$ we obtain

$$
\begin{aligned}
\mathcal{F}\left({ }^{+} c_{0}^{0} * \tilde{h}^{+}+{ }^{-} c_{0}^{0} * \tilde{h}^{-}\right)(\omega) & ={ }^{+} \hat{c}_{0}^{0}(\omega) \hat{\tilde{h}}^{+}(\omega)+{ }^{-} \hat{c}_{0}^{0}(\omega) \hat{\tilde{h}}^{-}(\omega) \\
& =\hat{c}_{0}^{0}(\omega)[\hat{h}(\omega-\pi / 2) \hat{\tilde{h}}(\omega-\pi / 2)+\hat{h}(\omega+\pi / 2) \hat{\tilde{h}}(\omega+\pi / 2)] \\
& \stackrel{(13)}{=} \quad 2 \hat{c}_{0}^{0}(\omega)
\end{aligned}
$$

since $\hat{h}$ and $\hat{\tilde{h}}$ are $2 \pi$-periodic. Therefore we have

$$
c_{0}^{0}=\frac{1}{2}\left({ }^{+} c_{0}^{0} * \tilde{h}^{+}+{ }^{-} c_{0}^{0} * \tilde{h}^{-}\right) .
$$

To close this Section, we give an overview of the whole signal processing routine in Figure 9 involving an FSWPT, filtering of the wavelet coefficients and an inverse FSWPT.

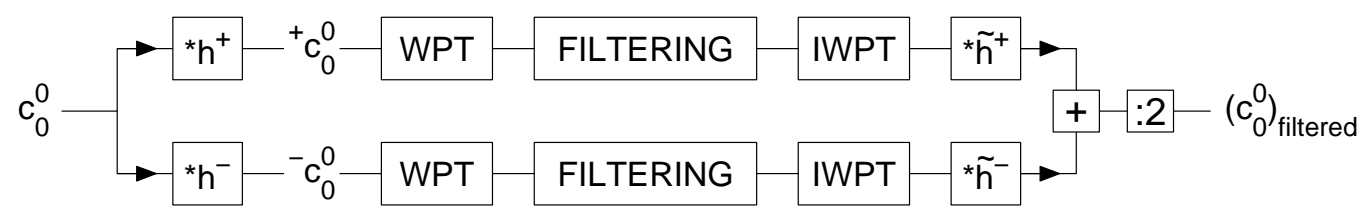

Figure 9. The whole clutter removal process from a signal $c_{0}^{0}$ consists of a separation into progressive and regressive components, two wavelet packet transforms (WPT), filtering, two inverse wavelet packet transforms (IWPT) and the synthesis of the filtered signal $\left(c_{0}^{0}\right)_{\text {filtered }}$. 


\section{FILTERING}

The main difference between clear-air signal and intermittent clutter like airplane or bird echos is that the former is stationary, i.e. its average frequency is time-independent, and the latter is transient. Hence, intermittent clutter can be seen as an inclined line in wavelet coefficient plots such as in Figure 8 (right). It can be well distinguished from the horizontal line representing the clear-air signal.

This difference is exploited in the filtering process. Each vector $c \in\left\{-c_{0}^{J}, \ldots,{ }^{-} c_{2}^{J}{ }_{-1},{ }^{+} c_{0}^{J}, \ldots,{ }^{+} c_{2^{J}-1}^{J}\right\}$ carries information of the signal at a certain well-localized frequency. Note that $c$ corresponds to a single row of the wavelet coefficient plot. Intermittent clutter will show up as a large and narrow peak in $\left(\left|c_{n}^{2}\right|^{2}\right)$. Clear-air signals as well as measurement noise will look like exponentially distributed random samples. ${ }^{8}$

To remove the peak, we estimate for each $c$ the expectation value $\frac{1}{\lambda}$ from (exponentially distributed) samples $\left|c_{n}\right|^{2}$ which do not belong to the peak, multiply $\frac{1}{\lambda}$ by some factor $q$ to achieve a $95 \%$ or $99 \%$ confidence and remove all samples greater that this threshold $s=\frac{q}{\lambda}$, i.e.

$$
\left(c_{n}\right)_{\text {filtered }}:= \begin{cases}c_{n} & \text { if }\left|c_{n}\right|^{2} \leq s \\ 0 & \text { if }\left|c_{n}\right|^{2}>s\end{cases}
$$

for all $n$. This type of wavelet filtering has been extensively studied by Donoho et al. , $^{10}$ See Figure 10 for an illustration of the thresholding process. We added some Gaussian distributed noise to the artificial RWP signal to make the example more instructive. Intermittent clutter has been completely removed and the clear-air signal has hardly been touched. The powerspectrum of the reconstructed filtered signal would show a clear peak at Doppler frequency.

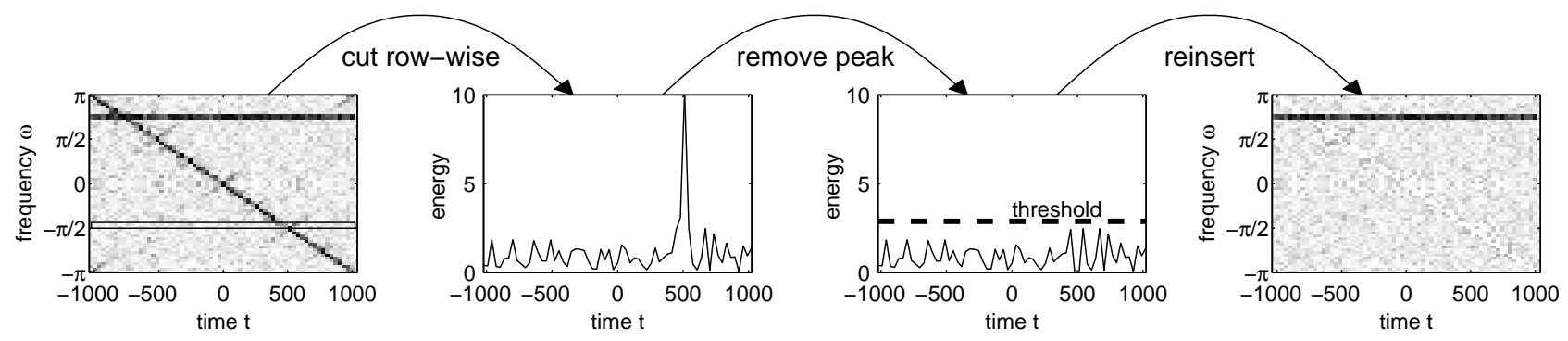

Figure 10. Intermittent clutter filtering of the artificial RWP signal plus some noise. Each row of the wavelet transform is filtered separately by thresholding. The threshold is selected adaptively from the coefficients which do not belong to a peak. Note that the clear-air signal is not influenced by this kind of thresholding.

Rows $c$ with stationary components such as clear-air signal and ground clutter are not influenced since the threshold $s$ will be much higher here. Only removal of peaks carrying both clear-air and intermittent clutter information influences the clear-air signal. However, the occurrence of such peaks (boxes III in the plots) is kept to a minimum by construction of the wavelet transform.

\section{RESULTS}

The special wavelet decomposition and filtering we have developed has proved to remove intermittent clutter from an artificial RWP signal very efficiently. Let us now consider a real RWP signal.

The signal as shown in Figure 11 (left) has been recorded by the German weather service with a $482 \mathrm{MHz}$ profiler in Lindenberg, Germany, at January 19, 1999. It is polluted by a strong airplane echo. The powerspectrum is completely useless for moment estimation in this case (Figure 11 (right)). For simplicity we assume the time spacing between samples to be one such that the frequency axis in the powerspectrum ranges from $\omega=-\pi$ to $\omega=\pi$. This is just a matter of axis scales - the filtering process is completely independent of the time spacing.

Figure 12 shows a plot of the FSWPT wavelet coefficients before (left) and after (right) filtering. We used Symlet sym10 wavelet filters of length 20 since we found that longer filters reduce artifacts in TF decompositions. 
The airplane echo looks very similar to the linear chirp in our simulated signal and has been successfully removed by the thresholding routine. The clear-air signal has been left nearly untouched except of a narrow region where it overlapped with clutter. Plots of the cleaned signal and powerspectra before and after clutter removal are shown in Figure 13. The cleaned powerspectrum is suitable for moment estimation without any further pre-processing.

Another example is given in Figures 14- 16. Although the signal-to-noise ratio is very low, the slowly varying airplane echo has been completely removed without influencing the clear-air signal. We have chosen this example to demonstrate that the DWT works considerably less well here. Figure 17 gives a plot of the cleaned DWT wavelet coefficients (left) and powerspectra of the original signal (solid) and the DWT cleaned signal (dotted). No matter how we choose the threshold, the DWT always gives unsatisfying results. Although the airplane echo has been partially removed, there are still several peaks in the powerspectrum leading to a wrong moment estimation.
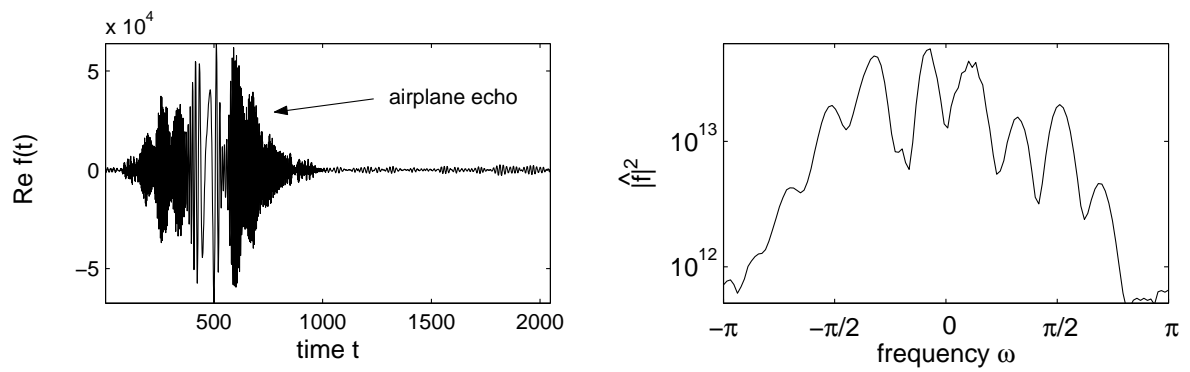

Figure 11. Left: Real part of a signal which is contaminated by intermittent clutter. Right: The corresponding averaged powerspectrum is completely useless. No clear-air peak is visible.
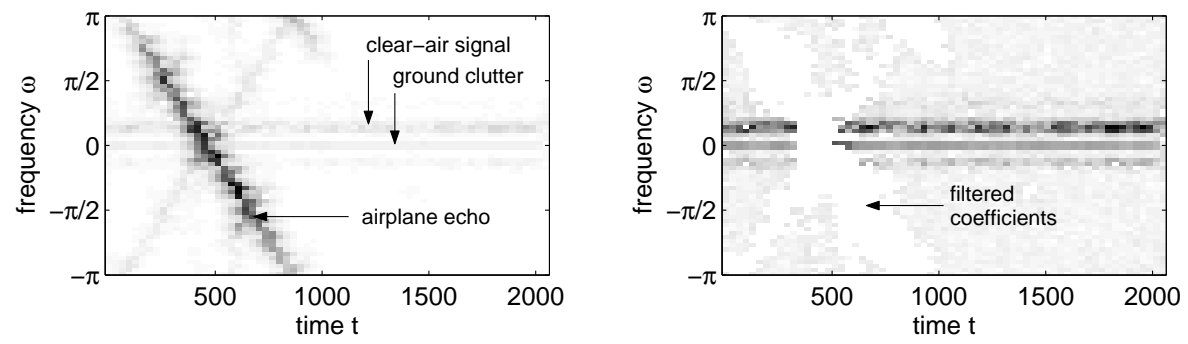

Figure 12. Left: FSWPT wavelet coefficients using Symlet sym10 wavelet filters. An airplane is visible as inclined line. Weak ground clutter and clear-air signal can be seen as horizontal lines. Right: Cleaned wavelet coefficients. The airplane echo has been completely removed.
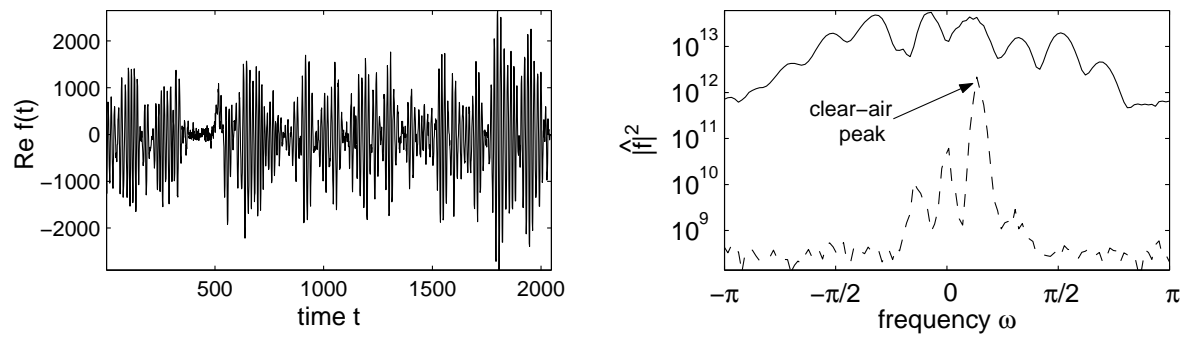

Figure 13. Left: Real part of the cleaned signal. To the human eye no clutter is visible anymore. Right: Powerspectra of the original signal (solid) and the cleaned signal (dashed). The clear-air peak can be easily detected in the cleaned powerspectrum. 


\section{CONCLUSIONS}

Based on existing wavelet algorithms for clutter removal we have introduced a new type of redundant timefrequency decomposition involving a wavelet packet decomposition combined with a band-pass filter to separate progressive and regressive signal parts. With this technique we are able to distinguish clutter from clear-air components very precisely. Only very few wavelet coefficients contain information of both components. A hard thresholding routine is applied to remove intermittent clutter. Since the wavelet coefficients are very well localized in frequency, transient signals appear as narrow peaks and can be easily removed by this method.

Although we have not investigated ground clutter filtering yet, there is definitely a great potential. Ground clutter always appears around zero frequency and can hence be detected and filtered if it does not mix with the clear-air signal. If it does, it is a-priori unclear how to separate them. Reflections from stationary objects like buildings do not produce a transient signal. We do not see the point in detecting stationary signals with wavelet techniques. This is not what they were build for. Fourier techniques seem more appropriate to us since they offer the best frequency localization at the expense of no time localization.

Wavelet algorithms are known for their high numerical efficiency. A DWT only requires $O(N)$ operations, where $N$ is the signal length. Our decomposition needs about 12 times more operations $(2 N$ for frequency separation, $5 \mathrm{~N}$ for each of the two level 5 wavelet packet transforms). Modern PCs with CPUs above $1 \mathrm{GHz}$ clock frequency can handle these computations in real-time.

We are currently receiving test data from a new Scintec profiler at Frankfurt airport, where a rather high percentage of data is contaminated by airplane clutter. The algorithms are currently being implemented in $\mathrm{C}++$ and will soon be tested on this data.

\section{ACKNOWLEDGMENTS}

This project is funded by the European Union in the framework of an EU CRAFT project named MEPROS (www.mepros.de). Gerd Teschke gratefully acknowledges support by DFG grant Te 354/1-2.

\section{REFERENCES}

1. J. Wilczak, R. Strauch, F. Ralph, B. Weber, D. Merritt, J. Jordan, D. Wolfe, L. Lewis, D. Wuertz, J. Gaynor, S. McLaughlin, R. Rogers, A. Riddle, and T. Dye, "Contamination of wind profiler data by migrating birds: Characteristics of corrupted data and potential solutions," J. Atmos. Oceanic Technol. 12, pp. 449-467, March 1995.

2. J. R. Jordan, R. J. Lataitis, and D. A. Carter, "Removing ground and intermittent clutter contamination from wind profiler signals using wavelet transforms," J. Atmos. Oceanic Technol. 14, pp. 1280-1297, December 1997.

3. J.-C. Boisse, V. Klaus, and J.-P. Aubagnac, "A wavelet transform technique for removing airplane echos from ST radar signals," J. Atmos. Oceanic Technol. 16, pp. 334-346, March 1999.

4. V. Lehmann and G. Teschke, "Wavelet based methods for improved wind profiler signal processing," Ann. Geophysicae 19, pp. 825-836, 2001.

5. G. Teschke, Waveletkonstruktion ueber Unschaerferelationen und Anwendungen in der Signalanalyse. PhD thesis, University of Bremen, 2001.

6. S. Mallat, A Wavelet Tour of Signal Processing, Academic Press, San Diego, CA, USA, 1999.

7. F. C. A. Fernandes, R. v. Spaendonck, M. J. Coates, and S. Burrus, "Directional Complex-Wavelet Processing," Proceedings of Society of Photo-Optical Instrumental Engineers-SPIE2000, Wavelet Applications in Signal Processing VIII, San Diego., 2000.

8. D. S. Zrnic, "Simulation of weatherlike doppler spectra and signals," J. Appl. Meteor. 14, 1975.

9. D. Donoho and I. Johnstone, "Minimax estimation via wavelet shrinkage," Tech. Rep. 402, Dept. of Statistics, Stanford University, 1992.

10. D. Donoho, I. Johnstone, G. Kerkyacharian, and D. Picard, "Density estimation by wavelet thresholding," tech. rep., Dept. of Statistics, Stanford University, 1993. 

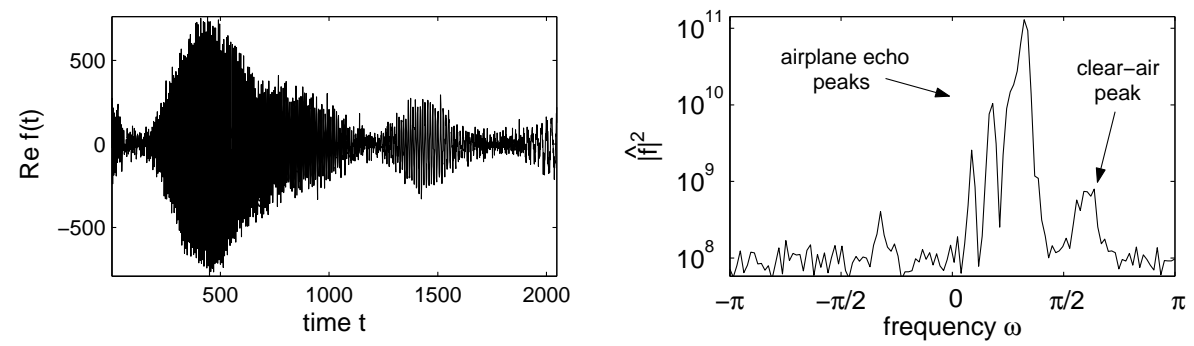

Figure 14. Left: Real part of another signal which is contaminated by slowly varying intermittent clutter. Right: The corresponding averaged powerspectrum contains a clutter peak which is more than $20 \mathrm{db}$ higher than the clear-air peak. Usual moment estimation techniques would fail here.
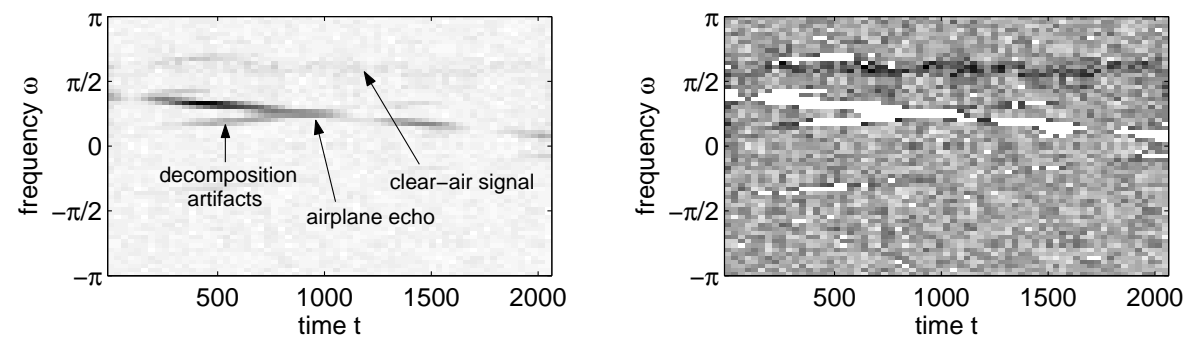

Figure 15. Left: FSWPT wavelet coefficients of the second example using Symlet sym10 wavelet filters. An airplane is visible as inclined line. The clear-air signal appears as faint horizontal line above $\pi / 2$. Right: Cleaned wavelet coefficients. The airplane echo has been completely removed.
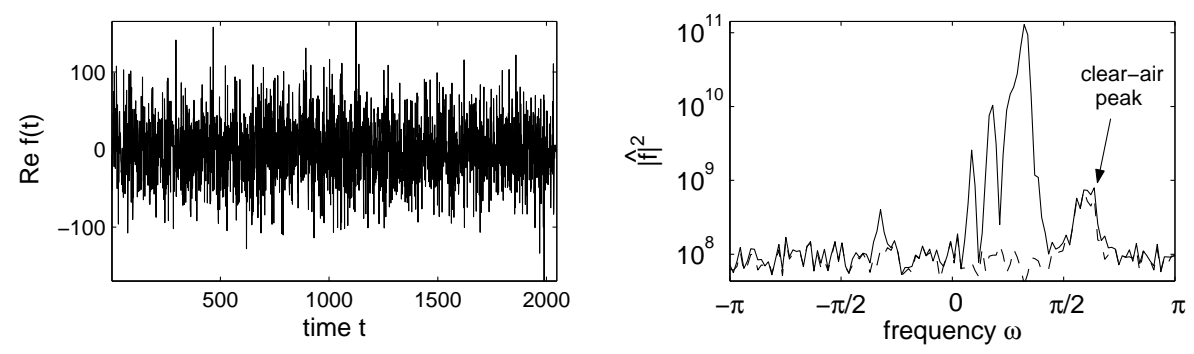

Figure 16. Left: Real part of the cleaned signal. Right: Powerspectra of the original signal (solid) and the cleaned signal (dashed). The three clutter peaks have been completely removed and the clear-air peak is left untouched.
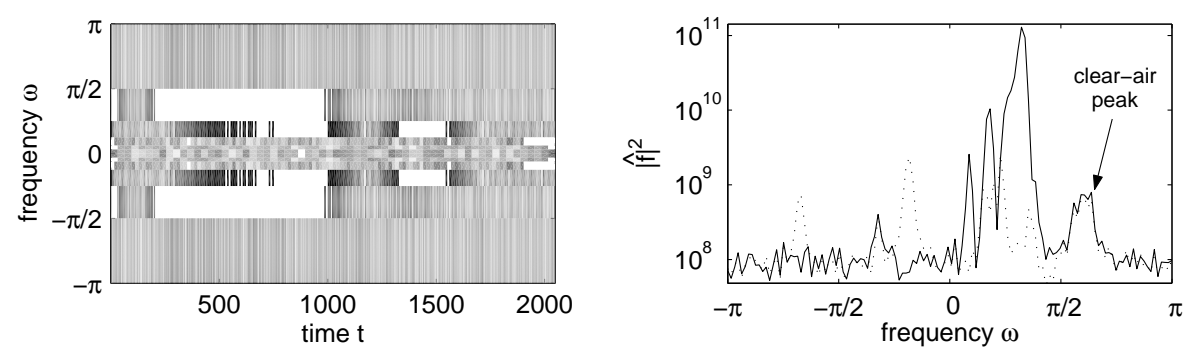

Figure 17. Left: Cleaned DWT wavelet coefficients of the second example using Symlet sym10 wavelet filters. The clear-air signal is not visible in this plot and the actual structure of the TF behavior is hidden. Right: Powerspectra of the original signal (solid) and the DWT cleaned signal (dotted). Clutter power has been reduced. However, several peaks of size comparable to the clear-air peak prevent a moment estimation. 